\author{
P. Pajunen • H. Koukkunen • M. Ketonen · T. Jerkkola $・$ P. Immonen-Räihä • \\ P. Kärjä-Koskenkari · K. Kuulasmaa · P. Palomäki · J. Mustonen · A. Lehtonen • \\ M. Arstila $\cdot$ T. Vuorenmaa $\cdot$ S. Lehto $\cdot$ H. Miettinen $\cdot$ J. Torppa $\cdot$ \\ J. Tuomilehto $\cdot$ Y. A. Kesäniemi $\cdot$ K. Pyörälä $\cdot$ V. Salomaa
}

\title{
Myocardial infarction in diabetic and non-diabetic persons with and without prior myocardial infarction: the FINAMI Study
}

Received: 18 June 2005 / Accepted: 22 July 2005 / Published online: 25 October 2005

C) Springer-Verlag 2005

\begin{abstract}
Aims/hypothesis: We compared the risk of acute coronary events in diabetic and non-diabetic persons with and without prior myocardial infarction (MI), stratified by age and sex. Methods: A Finnish MI-register study known as FINAMI recorded incident MIs and coronary deaths $(n=6988)$ among people aged 45 to 74 years in four areas of Finland between 1993 and 2002. The population-based FINRISK surveys were used to estimate the numbers of persons with prior diabetes and prior MI in the population. Results: Persons with diabetes but no prior MI and persons with prior MI but no diabetes had a markedly greater risk of a coronary event than persons
\end{abstract}

\footnotetext{
P. Pajunen $\cdot$ K. Kuulasmaa $\cdot$ J. Torppa

J. Tuomilehto $\cdot$ V. Salomaa $(\bowtie)$

KTL-National Public Health Institute,

Department of Epidemiology and Health Promotion,

Mannerheimintie 166,

FIN-00300, Helsinki, Finland

e-mail: veikko.salomaa@ktl.fi

Tel.: +358-9-47448620

Fax: +358-9-47448338

H. Koukkunen · S. Lehto · H. Miettinen · K. Pyörälä Kuopio University Hospital,

Kuopio, Finland
}

M. Ketonen · P. Palomäki · J. Mustonen

Central Hospital of North Karelia,

Joensuu, Finland

T. Jerkkola · Y. A. Kesäniemi

Oulu University Hospital and Biocenter Oulu,

Oulu, Finland

P. Immonen-Räihä · M. Arstila · T. Vuorenmaa

Turku University Hospital,

Turku, Finland

P. Kärjä-Koskenkari

Oulu City Hospital,

Oulu, Finland

A. Lehtonen

Turku City Hospital,

Turku, Finland without diabetes and without prior MI. The rate of recurrent MI among non-diabetic men with prior MI was higher than the incidence of first MI among diabetic men aged 45 to 54 years. The rate ratio was 2.14 (95\% CI 1.40 3.27 ) among men aged 50. Among elderly men, diabetes conferred a higher risk than prior MI. Diabetic women had a similar risk of suffering a first MI as non-diabetic women with a prior MI had for suffering a recurrent MI. Conclusions/interpretation: Both persons with diabetes but no prior MI, and persons with a prior MI but no diabetes are high-risk individuals. Among men, a prior MI conferred a higher risk of a coronary event than diabetes in the 45-54 year age group, but the situation was reversed in the elderly. Among diabetic women, the risk of suffering a first MI was similar to the risk that non-diabetic women with prior MI had of suffering a recurrent MI.

Keywords Coronary disease $\cdot$ Diabetes Myocardial infarction

Abbreviations MI: myocardial infarction - MONICA: Monitoring Trends and Determinants of Cardiovascular Disease

\section{Introduction}

Worldwide the number of people diagnosed with diabetes mellitus is increasing rapidly [1]. Diabetes is associated with a two- to four-fold increase in the risk of developing $\mathrm{CHD}$ [2], and cardiovascular diseases are the major cause of death among diabetic persons [3].

It remains controversial whether diabetic persons have a similar risk of developing acute CHD events as non-diabetic patients who have suffered a prior myocardial infarction (MI) [4-12]. An earlier study from Finland suggested that type 2 diabetic patients have a risk equal to that in nondiabetic patients with a prior history of MI [4]. This study has influenced current prevention guidelines in several countries $[13,14]$. However, several other studies have reported contrasting results $[5,6,9,11,12]$ and some found 
that diabetic women are at particularly high risk [5, 10]. Since the question is of considerable clinical importance, we compared in the present study the rate of coronary events in diabetic and non-diabetic men and women with and without a prior MI in different age groups in a population-based setting in Finland between 1993 and 2002.

\section{Subjects and methods}

Study population The numerators for the coronary event rates were obtained from a population-based MI register, FINAMI [15]. For the denominators, full population counts of the study years and study areas were obtained in 5-year age groups from the National Population Register, and these were divided according to diabetes and history of prior MI status using prevalence estimates from the FINRISK population surveys [16]. These surveys have been carried out every 5 years since 1972 (since 1997 in Oulu) to assess cardiovascular risk factor levels in Finland [16]. Survey participants aged 45-74 years from the years 1992, 1997 and 2002 were included in the current analyses. The surveys also cover some of the surroundings of the FINAMI areas. The FINRISK questionnaire included a question: 'Has a doctor ever told you that you have (1) diabetes, or (2) latent diabetes?' In addition, record linkage of the study data with the computerised records of the National Social Insurance Institution was used to identify persons who had received reimbursements for antidiabetic medications. The record linkage was based on the individual identification code, which is unique to every resident of Finland. A diabetic person receives his/her antidiabetic medications free of charge in Finland. A detailed written statement from a physician in charge of the treatment verifying the diagnosis of diabetes is needed to obtain the right to free-of-charge antidiabetic medication.

All first-ever MIs and first recurrent (=the 2nd) MIs and coronary deaths among persons aged $45-74$ years between 1993 and 2002 were selected from the FINAMI register $(n=6,988)$. The register covers the following four areas in Finland: the city of Turku in south-western Finland, the city of Oulu in north-western Finland, the city of Kuopio in eastern Finland, the city of Joensuu, and some surrounding rural areas in eastern Finland. For the city of Oulu there were data for the years 1993, 1997, 1999, 2001 and 2002, whereas for the other areas data were available for the whole 10-year period of 1993-2002, except for Turku for the year 1999 and Kuopio for the year 1998. In the FINAMI study, trained nurses supervised by register physicians were responsible for data collection from medical records, death certificates and autopsy reports [15, 17]. The FINAMI data were also linked to the Social Insurance Institution's drug reimbursement records. In the FINAMI study a person was considered as diabetic if the register nurse found the diagnosis of diabetes from medical records or if the person had received reimbursements for antidiabetic medication. Out of 6,988 persons in the FINAMI register, 1,674 (24\%) had diabetes. No information on the duration of diabetes was collected in the FINAMI register.
The time-frame for one event in the FINAMI register was 28 days according to the practice of the WHO Monitoring Trends and Determinants of Cardiovascular Disease (MONICA) Project [18]. The event was taken as the first for a particular person if there were no data on a previous, clinically recognised MI, and as recurrent if evidence of a prior MI was found from the hospital records.

Both the FINAMI and FINRISK studies have been approved by the Ethical Committee of the National Public Health Institute and were carried out in accordance with the Declaration of Helsinki as revised in 2000. Participants of the FINRISK study gave informed consent. The FINAMI study was carried out by reviewing the hospital documents and using record linkage techniques without any contact with the patients, inline with legal and ethical requirements.

Statistical analysis In the main analyses we considered as diabetic those persons who reported in the FINRISK questionnaire that they had diabetes and/or who had received the right for free-of-charge antidiabetic medications. In this analysis persons reporting latent diabetes in the questionnaire were taken to be non-diabetic. To evaluate the impact of the definition and duration of diabetes we repeated the analyses by including in the diabetic group also persons reporting latent diabetes in the questionnaire.

Poisson regression modelling of pooled data from the FINRISK surveys in 1992, 1997 and 2002 was used to estimate the prevalence of diabetes and previous history of MI for each 5-year age group in each calendar year. The log-linear model included linear and quadratic effects of age group and calendar year and dummy covariates for east/west Finland and the FINAMI area/its neighbourhood. The prevalence estimates were applied to the official population figures to obtain denominators for the event rates. Poisson regression models were also used for calculating age-specific rates of first-ever MI events in diabetic persons and first recurrent (=the 2nd) MI events in non-diabetic patients. The different groups were compared using rate ratios and $95 \%$ CIs. Since there was an interaction between the study year and the diabetes group, the results are calculated for the year 1997, which was in the middle of our study period.

CHD event and mortality rates, age-standardised using weights derived from the combined age-distribution of MI and stroke patients in the WHO MONICA Project [18],

Table 1 Numbers of first and first recurrent $(=2$ nd) myocardial infarction (MI) events in diabetic (DM+) and non-diabetic (DM-) persons aged 45-74 years in the FINAMI register between 1993 and 2002

\begin{tabular}{lrllr}
\hline & \multicolumn{2}{l}{ First MI } & & \multicolumn{2}{l}{ Recurrent MI } \\
\cline { 2 - 3 } \cline { 5 - 6 } & DM+ & DM- & DM+ & \multicolumn{1}{l}{ DM- } \\
\hline Men & $659(19 \%)$ & $2757(81 \%)$ & $401(27 \%)$ & $1082(73 \%)$ \\
Women & $406(26 \%)$ & $1182(74 \%)$ & $208(42 \%)$ & $293(58 \%)$ \\
Total & $1065(21 \%)$ & $3939(79 \%)$ & $609(31 \%)$ & $1375(69 \%)$ \\
\hline
\end{tabular}


Table 2 Numbers of diabetic $(\mathrm{DM}+)$ and non-diabetic (DM-) persons and persons with a prior myocardial infarction (MI) aged 45-74 years in the FINRISK population surveys in 1992 , 1997 and 2002

\begin{tabular}{lrrcrc}
\hline & DM- MI- & DM+, MI- & DM-, MI+ & DM+, MI+ & Total \\
\hline DM & & & & & \\
Men, total & $6139(89 \%)$ & $305(4.4 \%)$ & $440(6.3 \%)$ & $65(0.94 \%)$ & 6949 \\
45-54 years & $2501(95 \%)$ & $79(3.0 \%)$ & $54(2.0 \%)$ & $9(0.34 \%)$ & 2643 \\
55-64 years & $2504(87 \%)$ & $141(4.9 \%)$ & $206(7.2 \%)$ & $30(1.0 \%)$ & 2881 \\
65-74 years & $1134(80 \%)$ & $85(6.0 \%)$ & $180(13 \%)$ & $26(1.8 \%)$ & 1425 \\
Women, total & $6562(95 \%)$ & $220(3.2 \%)$ & $119(1.7 \%)$ & $26(0.38 \%)$ & 6927 \\
45-54 years & $2864(98 \%)$ & $54(1.8 \%)$ & $15(0.51 \%)$ & $0(0 \%)$ & 2933 \\
$55-64$ years & $2852(95 \%)$ & $95(3.2 \%)$ & $58(1.9 \%)$ & $14(0.46 \%)$ & 3019 \\
65-74 years & $846(87 \%)$ & $71(7.3 \%)$ & $46(4.7 \%)$ & $12(1.2 \%)$ & 975 \\
DM (including latent DM) & & & & \\
Men, total & $5985(86 \%)$ & $459(6.6 \%)$ & $414(6.0 \%)$ & $91(1.3 \%)$ & 6949 \\
$45-54$ years & $2461(93 \%)$ & $119(4.5 \%)$ & $53(2.0 \%)$ & $10(0.38 \%)$ & 2643 \\
$55-64$ years & $2414(84 \%)$ & $231(8.0 \%)$ & $192(6.7 \%)$ & $44(1.5 \%)$ & 2881 \\
$65-74$ years & $1110(78 \%)$ & $109(7.7 \%)$ & $169(12 \%)$ & $37(2.6 \%)$ & 1425 \\
Women, total & $6375(92 \%)$ & $407(5.9 \%)$ & $109(1.6 \%)$ & $36(0.52 \%)$ & 6927 \\
$45-54$ years & $2798(95 \%)$ & $120(4.1 \%)$ & $14(0.48 \%)$ & $1(0.03 \%)$ & 2933 \\
$55-64$ years & $2761(91 \%)$ & $186(6.2 \%)$ & $52(1.7 \%)$ & $20(0.66 \%)$ & 3019 \\
$65-74 y e a r s$ & $816(84 \%)$ & $101(10 \%)$ & $43(4.4 \%)$ & $15(1.5 \%)$ & 975 \\
\hline
\end{tabular}

were expressed per 100,000 persons per year. Age-standardised event rates were reported separately for three groups: 'Diabetes, no prior MI', 'No diabetes, no prior MI' and 'No diabetes, prior MI'. The group 'Diabetes, prior MI' was not reported because of low precision caused by a small number of such persons in the FINRISK survey. The 95\% CIs for the incidence, recurrence and mortality rates were calculated considering the sampling variation of both the numerator and denominator, using the Taylor approximation. The statistical analyses were carried out using software from the SAS institute [19]. We considered $p$ values smaller than 0.05 to be statistically significant.

\section{Results}

In the FINAMI register, $19 \%$ of men and $26 \%$ of women suffering their first MI had diabetes. The respective percentages for recurrent MIs were $27 \%$ among men and $42 \%$ among women (Table 1). Based on the FINRISK survey $(n=13,876)$, the estimated prevalence of diabetes in the general population aged $45-74$ years was 5.3\% among men and 3.6\% among women. The estimated prevalence increased with age and was highest among the oldest age group (65-74 years), namely 7.5\% among men and $8.9 \%$ among women. Table 2 shows prevalences of diabetic and non-diabetic participants and participants with a prior MI in
Table 3 Incidence and mortality rates per 100,000 of first-ever myocardial infarction (MI) events among non-diabetic persons (DM-) and the rate ratios of first MI among diabetic patients $(\mathrm{DM}+)$ and first recurrent $\mathrm{MI}$ among non-diabetic patients, compared with the first category, by age and sex

Values are given with $95 \%$ CIs. Pooled MI event rate is also shown for comparison.

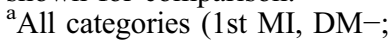
1st MI, DM+; 2nd MI, DM-; and 2 nd MI, DM+) pooled

\begin{tabular}{|c|c|c|c|c|}
\hline & $\begin{array}{l}\text { Incidence rate } \\
1 \text { st MI, DM- } \\
\text { (reference category) }\end{array}$ & $\begin{array}{l}\text { Rate ratio } \\
1 \text { st } \mathrm{MI}, \mathrm{DM}+\end{array}$ & $\begin{array}{l}\text { Rate ratio } \\
\text { 2nd MI, DM- }\end{array}$ & Pooled rates ${ }^{\mathrm{a}}$ \\
\hline \multicolumn{5}{|l|}{ Morbidity } \\
\hline \multicolumn{5}{|l|}{ Men } \\
\hline $45-54$ years & $260(239-282)$ & $4.4(3.2-5.7)$ & $11(7.8-14)$ & $364(339-389)$ \\
\hline 55-64 years & $563(526-601)$ & $5.1(4.2-6.0)$ & $5.7(4.7-6.7)$ & 997 (947-1047) \\
\hline $65-74$ years & 915 (859-971) & $6.3(5.2-7.5)$ & $4.6(3.7-5.4)$ & $2102(2018-2186)$ \\
\hline \multicolumn{5}{|l|}{ Women } \\
\hline $45-54$ years & $39(31-47)$ & $15(5.9-24)$ & $23(5.8-31)$ & $54(45-63)$ \\
\hline 55-64 years & $147(129-165)$ & $7.8(5.1-10)$ & $7.0(4.6-9.5)$ & $229(207-251)$ \\
\hline $65-74$ years & $454(420-489)$ & $6.8(4.8-8.8)$ & $5.0(3.8-6.1)$ & $872(828-916)$ \\
\hline \multicolumn{5}{|l|}{ Mortality } \\
\hline \multicolumn{5}{|l|}{ Men } \\
\hline $45-54$ years & $85(73-98)$ & $5.5(3.3-7.7)$ & $9.3(5.3-13)$ & $123(108-138)$ \\
\hline 55-64 years & $206(183-228)$ & $5.2(3.8-6.5)$ & $7.2(5.6-8.9)$ & $338(357-419)$ \\
\hline $65-74$ years & $425(388-462)$ & $6.8(5.3-8.3)$ & $5.0(3.9-6.0)$ & $1013(954-1072)$ \\
\hline \multicolumn{5}{|l|}{ Women } \\
\hline $45-54$ years & Too few cases & Too few cases & Too few cases & $11(7-15)$ \\
\hline 55-64 years & $43(33-53)$ & $8.8(4.2-14)$ & $11(5.8-17)$ & $76(63-89)$ \\
\hline $65-74$ years & $184(163-205)$ & $7.2(4.8-9.5)$ & $5.2(3.7-6.7)$ & $359(331-387)$ \\
\hline
\end{tabular}




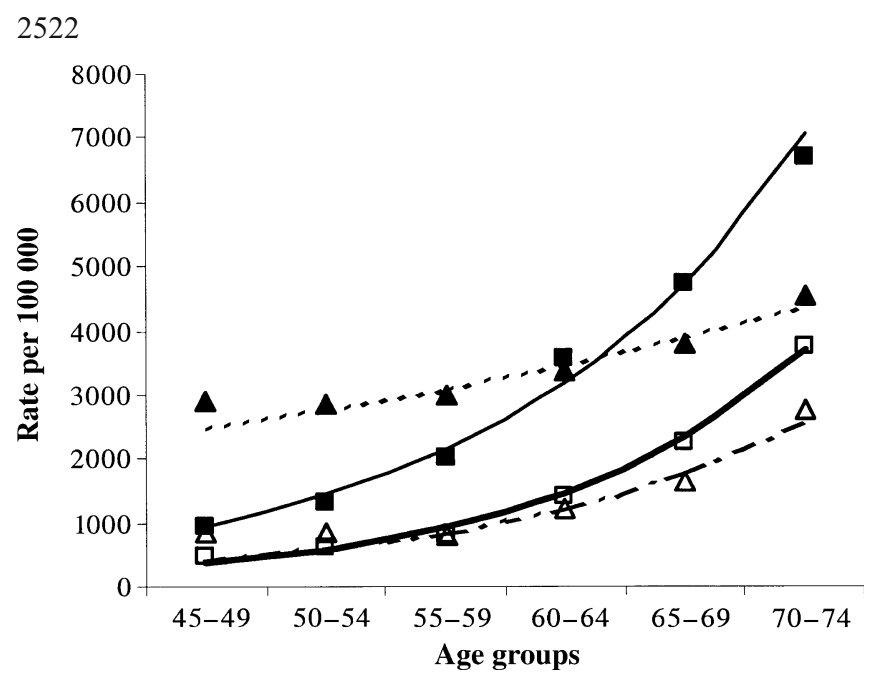

Fig. 1 Poisson regression model of morbidity. Rates of first MI events among diabetic men (filled squares), first recurrent $(=2 \mathrm{nd})$ MI among non-diabetic men (filled triangles), first MI events among diabetic women (open squares), and first recurrent MI among nondiabetic women (open triangles) by age group. The lines are based on Poisson regression modelling

the FINRISK population surveys, which were used for calculating the population denominators for the event rates.

The relationship between the incidence rate of first MI events among diabetic men and the rate of first recurrent (=2nd) MI events among non-diabetic men was significantly dependent on age $(p<0.001$ for interaction). The incidence of first $\mathrm{MI}$ events among diabetic men increased on average by $8.0 \%(95 \%$ CI $7.0-9.0 \%)$ per each year of age after the age of 45 , whereas the rate of recurrent MI events among non-diabetic men increased by $2.3 \%(1.4-$ $3.1 \%)$ per year. The effect of calendar year also approached statistical significance $(p=0.08)$. Therefore, the rest of the results are presented as age-specific rates and rate ratios estimated for the year 1997. Among women the aforementioned interactions were not statistically significant.

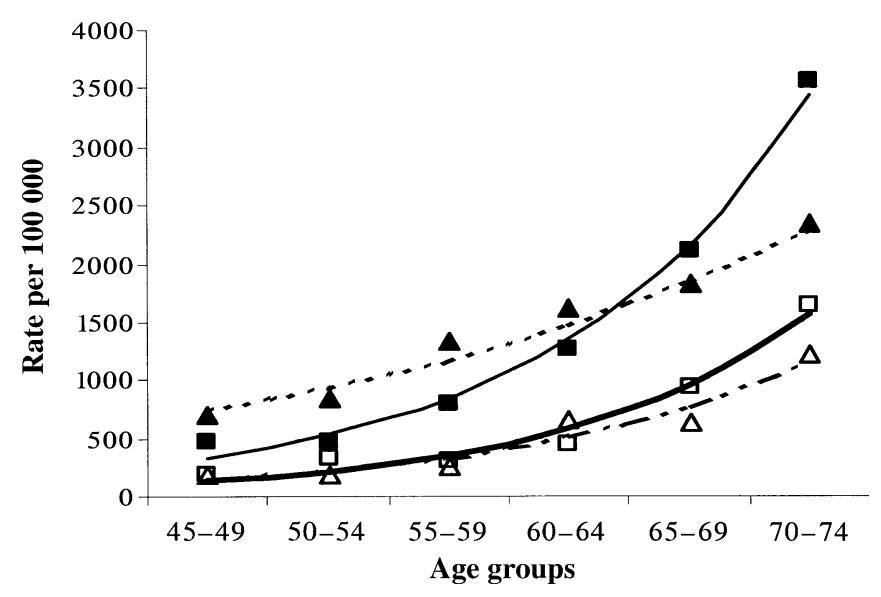

Fig. 2 Poisson regression model of mortality. Rates of first MI events among diabetic men (filled squares), first recurrent $(=2 \mathrm{nd})$ MI among non-diabetic men (filled triangles), first MI events among diabetic women (open squares), and first recurrent MI among nondiabetic women (open triangles) by age group. The lines are based on Poisson regression modelling
The MI event rates were 4-15 times higher, depending on age group and sex, in persons with diabetes but no prior MI and 5-23 times higher in persons with a prior MI but no diabetes than in persons without either diagnosis (Table 3 ). The rate of recurrent MI among non-diabetic men with a prior history of MI was higher than the incidence of first MI among diabetic men under 55 years of age (Table 3 ). The rate ratio (rate of a recurrent MI among non-diabetic men/ incidence rate of the first MI among diabetic men) was 2.14 $(95 \%$ CI 1.40-3.27) at the age of 50 years. In the 55-64-year age group, diabetic men had a similar risk of first MI as nondiabetic men with a prior MI had of their first recurrent (=2nd) MI (Table 3 ). The regression curves were crossed at the age of 64 years (Fig. 1). In the elderly, diabetes conferred a higher risk of MI than a prior MI; the rate ratio among men at the age of 70 was 0.68 (95\% CI 0.49-0.94).

Diabetic women had a similar risk of their first MI as non-diabetic women with a prior MI had of their first recurrent (=2nd) MI (Table 3 and Fig. 1). In women older

Table 4 Incidence and mortality rates, and rate ratios as in Table 3, but with latent diabetes included in the diabetic group

\begin{tabular}{|c|c|c|c|}
\hline & $\begin{array}{l}\text { Incidence per } 100,000 \\
1 \text { st MI, DM- } \\
\text { (reference category) }\end{array}$ & $\begin{array}{l}\text { Rate ratio } \\
1 \text { st MI, DM+ }\end{array}$ & $\begin{array}{l}\text { Rate ratio } \\
\text { 2nd MI, DM- }\end{array}$ \\
\hline \multicolumn{4}{|c|}{ Morbidity } \\
\hline \multicolumn{4}{|l|}{ Men } \\
\hline $\begin{array}{r}45-54 \\
\text { years }\end{array}$ & $265(243-287)$ & $2.8(2.0-3.6)$ & $11(8.2-14)$ \\
\hline $\begin{array}{r}55-64 \\
\text { years }\end{array}$ & $582(543-620)$ & $3.0(2.5-3.6)$ & $5.8(4.9-6.8)$ \\
\hline $\begin{array}{r}65-74 \\
\text { years }\end{array}$ & 935 (878-992) & $4.3(3.5-5.1)$ & $4.7(4.0-5.5)$ \\
\hline \multicolumn{4}{|l|}{ Women } \\
\hline $\begin{array}{r}45-54 \\
\text { years }\end{array}$ & $40(32-48)$ & $5.5(2.3-8.8)$ & $26(7.2-44)$ \\
\hline $\begin{array}{l}55-64 \\
\text { years }\end{array}$ & $152(134-171)$ & $3.5(2.3-4.8)$ & $7.6(5.1-10)$ \\
\hline $\begin{array}{r}65-74 \\
\text { years }\end{array}$ & $473(437-509)$ & $3.9(2.7-5.1)$ & $5.1(4.1-6.1)$ \\
\hline \multicolumn{4}{|c|}{ Mortality } \\
\hline \multicolumn{4}{|l|}{ Men } \\
\hline $\begin{array}{r}45-54 \\
\text { years }\end{array}$ & $87(75-100)$ & $3.5(2.1-4.8)$ & $9.3(5.5-13)$ \\
\hline $\begin{array}{r}55-64 \\
\text { years }\end{array}$ & $212(189-236)$ & $3.0(2.2-3.8)$ & $7.4(5.8-9.1)$ \\
\hline $\begin{array}{r}65-74 \\
\text { years }\end{array}$ & $435(397-472)$ & $4.6(3.6-5.7)$ & $5.1(4.2-6.1)$ \\
\hline \multicolumn{4}{|l|}{ Women } \\
\hline $\begin{array}{c}45-54 \\
\text { years }\end{array}$ & Too few cases & Too few cases & Too few cases \\
\hline $\begin{array}{r}55-64 \\
\text { years }\end{array}$ & $45(35-55)$ & $4.0(1.9-6.1)$ & $12(6.4-18)$ \\
\hline $\begin{array}{r}65-74 \\
\text { years }\end{array}$ & $192(170-214)$ & $4.1(2.7-5.5)$ & $5.3(3.9-6.7)$ \\
\hline
\end{tabular}

Values are given with $95 \%$ CIs 
than 54 years the curves diverged (Fig. 1) so that in the older age groups diabetes conferred a higher risk of MI than prior MI, but the difference did not reach statistical significance. The rate ratio among women at age 70 years was 0.73 (95\% CI 0.46-1.18).

Mortality followed the same patterns as morbidity (Fig. 2). Mortality from a recurrent MI among non-diabetic men with a prior history of MI was higher than mortality from a first MI among diabetic men under 65 years of age (Fig. 2 and Table 4). The rate ratio at the age of 50 years among men was 1.92 (95\% CI 1.10-3.36). The mortality curves crossed at the age of 64 years among men and the rate ratio at age 70 years was 0.73 (95\% CI $0.50-1.06)$. Among women there were no statistically significant differences in mortality between non-diabetic patients with a prior MI and diabetic patients without prior MI in any of the age groups (Fig. 2 and Table 3 ).

In the sensitivity analysis, where persons with latent diabetes were included in the diabetic group in the population denominator, the event rates were lower, but the associations with age were similar to in the main analyses (Table 4).

\section{Discussion}

We investigated whether non-diabetic persons with a prior history of MI had a higher risk for a recurrent MI event than diabetic persons for their first MI and found that the results depended on sex and the age group studied. Among men, a prior history of MI conferred a higher risk than previously diagnosed diabetes until the age of 54 years, whereafter there was no difference between the risks conferred by diabetes or previous MI until the age of 70 years. After the age of 70, diabetes conferred a higher risk than prior history of MI. Among women, the risk of a recurrent MI in nondiabetic women with a prior MI was similar to that of the first MI of diabetic women in all age groups. Thus, the discrepancy in earlier studies on this issue may be partly explained by the different age and sex distribution of study participants. It should be noted, however, that both persons with diabetes but no prior MI and persons with a prior MI but no diabetes had much higher MI event rates than persons without diabetes and prior MI.

In the study by Haffner et al. [4], with 7 years of followup, the mean age of the study population at baseline was less than 60 years, whereas in many other studies the mean age of the study population was $60-65$ years at baseline [6, $7,9]$. In these age groups the risk in the present study was equal in the diabetic (for first MI) and non-diabetic subjects (for recurrent MI) also among men. Vaccaro et al. [12] observed in the MRFIT follow-up study that even though prior MI conferred a higher risk of CHD death than diabetes, the hazard ratios comparing prior MI with diabetes declined with increasing age. In the US Physicians' Health Study, the relative risk of death from CHD was higher in non-diabetic patients with prior MI than that in diabetic patients with no prior CHD. This finding held true across all age groups; however, the difference between the two risk groups attenuated with increasing age [6]. In the Nurses' Health Study [5] the excess risk of CHD mortality associated with prior CHD was higher than that associated with diabetes in all age groups studied $(<55$ years, 5564 years and $\geq 65$ years). This was, however, dependent on the duration of diabetes so that in women with a diabetes duration of more than 15 years the risk of CHD death was similar to that in women with prior CHD but no diabetes.

It is well documented that diabetes raises the risk of CHD to a greater extent in women than in men [7, 10, 20 23]. It is as yet unknown what generates this increased risk in diabetic women that already exists in the prediabetic stages $[22,24]$ as it is only partly explained by an excess of the classic risk factors $[20,22]$. Our results are in agreement with some earlier studies which have observed that a history of MI and diabetes are associated with equal risks for acute cardiovascular events in women [7, 10]. However, in the study by Mukamal et al. [7] the results were sensitive to the duration of diabetes; when newly detected cases of diabetes were added to the analysis, CHD conferred a higher risk than diabetes. Women are actively screened for impaired glucose tolerance and diabetes during pregnancy, and thus report latent diabetes more often than men, a fact that was seen also in this study.

The strength of the present study is that it is a large population-based study. To assess the sensitivity of our analyses to the definition of diabetes, the analysis was carried out twice, including and excluding persons with latent diabetes in the diabetic population of the FINAMI areas. This had no effect on our main findings regarding the effects of age and sex.

The study had some obvious limitations that should be taken into account. The prevalence of diabetes and a history of MI was estimated from a population survey. Slight variation in the sampling fractions between the study regions was not taken into account and one of them (Oulu) had only been surveyed since 1997 . This approximation should not have a substantial effect on the findings. The diagnosis of diabetes and prior MI were not based on exactly the same criteria in the FINRISK population surveys and in the FINAMI register. In the FINRISK surveys self-reported data, verified and complemented with the drug-reimbursement register, were used. In the FINAMI register, however, the diagnoses were based on the review of hospital documents, death certificates and autopsy reports, again complemented with the drug-reimbursement register. While self-reported history of MI is usually fairly reliable, the self-reported history of diabetes is known to substantially underestimate the real prevalence of diabetes. On the other hand, recent studies have shown that glucose tolerance tests reveal a substantial amount of new cases of diabetes and impaired glucose tolerance among patients with MI $[25,26]$. During the period of our study, glucose tolerance tests were seldom performed on MI patients in Finnish hospitals. Only 56 new cases of diabetes were diagnosed during the treatment of MI. This amount is so small that their inclusion or exclusion in the analyses did not make any substantial difference. We could not examine the effects of the type and duration of diabetes on the risk of 
coronary events because our data collection did not include information about these aspects of diabetes. Indirectly, however, the fact that the effect of diabetes was diluted in analyses which also included latent diabetes, suggests that the risk of MI increases with the duration of diabetes. This interpretation is consistent with other literature [5].

In conclusion, both persons with diabetes but no prior MI and persons with a prior MI but no diabetes had a markedly higher risk of a coronary event than persons with no diabetes and no prior MI. Among men, a prior MI conferred a higher risk than diabetes in the 45-54 year age group, but in the elderly, the diabetic patients had a higher risk of developing their first MI than non-diabetic patients with a prior MI had of suffering their first recurrent MI. In diabetic women the risk of suffering a first MI was similar to the risk, in women with prior MI, of suffering a recurrent MI.

Acknowledgement The FINAMI Project is supported by the Finnish Foundation for Cardiovascular Research.

\section{References}

1. Zimmet P, Alberti KG, Shaw J (2001) Global and societal implications of the diabetes epidemic. Nature 414:782-787

2. Beckman JA, Creager MA, Libby P (2002) Diabetes and atherosclerosis: epidemiology, pathophysiology, and management. JAMA 287:2570-2581

3. Creager MA, Luscher TF, Cosentino F et al. (2003) Diabetes and vascular disease: pathophysiology, clinical consequences, and medical therapy: Part I. Circulation 108:1527-1532

4. Haffner SM, Lehto S, Rönnemaa T et al. (1998) Mortality from coronary heart disease in subjects with type 2 diabetes and in nondiabetic subjects with and without prior myocardial infarction. N Engl J Med 339:229-234

5. Hu FB, Stampfer MJ, Solomon CG et al. (2001) The impact of diabetes mellitus on mortality from all causes and coronary heart disease in women: 20 years of follow-up. Arch Intern Med 161:1717-1723

6. Lotufo PA, Gaziano JM, Chae CU et al. (2001) Diabetes and all-cause and coronary heart disease mortality among US male physicians. Arch Intern Med 161:242-247

7. Mukamal KJ, Nesto RW, Cohen MC et al. (2001) Impact of diabetes on long-term survival after acute myocardial infarction: comparability of risk with prior myocardial infarction. Diabetes Care 24:1422-1427

8. Evans JM, Wang J, Morris AD (2002) Comparison of cardiovascular risk between patients with type 2 diabetes and those who had had a myocardial infarction: cross-sectional and cohort studies. BMJ 324:939-942

9. Cho E, Rimm EB, Stampfer MJ et al. (2002) The impact of diabetes mellitus and prior myocardial infarction on mortality from all causes and from coronary heart disease in men. J Am Coll Cardiol 40:954-960

10. Becker A, Bos G, de Vegt F et al. (2003) Cardiovascular events in type 2 diabetes: comparison with nondiabetic individuals without and with prior cardiovascular disease. 10-year followup of the Hoorn Study. Eur Heart J 24:1406-1413
11. Lee CD, Folsom AR, Pankow JS et al. (2004) Atherosclerosis Risk in Communities (ARIC) Study Investigators. Cardiovascular events in diabetic and nondiabetic adults with or without history of myocardial infarction. Circulation 109:855-860

12. Vaccaro O, Eberly LE, Neaton JD et al. (2004) Multiple Risk Factor Intervention Trial Research Group. Impact of diabetes and previous myocardial infarction on long-term survival: $25-$ year mortality follow-up of primary screenees of the Multiple Risk Factor Intervention Trial. Arch Intern Med 164:14381443

13. Expert Panel on Detection, Evaluation, and Treatment of High Blood Cholesterol in Adults (2001) Executive summary of the third report of The National Cholesterol Education Program (NCEP) Expert Panel on Detection, Evaluation, and Treatment of High Blood Cholesterol In Adults (Adult Treatment Panel III). JAMA 285:2486-2497

14. Pearson TA, Blair SN, Daniels SR et al. (2002) AHA guidelines for primary prevention of cardiovascular disease and stroke: 2002 update: Consensus panel guide to comprehensive risk reduction for adult patients without coronary or other atherosclerotic vascular diseases. American Heart Association Science Advisory and Coordinating Committee. Circulation 106 388-391

15. Salomaa V, Ketonen M, Koukkunen H et al. (2003) Trends in coronary events in Finland during 1983-1997; the FINAMI study. Eur Heart J 24:311-319

16. Vartiainen E, Jousilahti P, Alfthan G et al. (2000) Cardiovascular risk factor changes in Finland, 1972-1997. Int J Epidemiol 29:49-56

17. Salomaa V, Ketonen M, Koukkunen H et al. (1996) Decline of coronary heart disease mortality in Finland during 1983 to 1992: roles of incidence, recurrence, and case-fatality. The FINMONICA MI Register Study. Circulation 94:3130-3137

18. Tunstall-Pedoe H (ed.) (2003) Prepared by Tunstall-Pedoe H, Kuulasmaa K, Tolonen H, Davidson M, Mendis S with 64 other contributors for The WHO MONICA Project. MONICA monograph and multimedia sourcebook. World Health Organization, Geneva

19. SAS Institute, Inc. (1999) Users guide, statistics, Version 8. Cary, NC

20. Howard BV, Cowan LD, Go O et al. (1998) Adverse effects of diabetes on multiple cardiovascular disease risk factors in women. The Strong Heart Study. Diabetes Care 21:1258-1265

21. DeStefano F, Ford ES, Newman J et al. (1993) Risk factors for coronary heart disease mortality among persons with diabetes. Ann Epidemiol 3:27-34

22. The DECODE Study Group (2003) Gender difference in allcause and cardiovascular mortality related to hyperglycaemia and newly diagnosed diabetes. Diabetologia 46:608-617

23. Juutilainen A, Kortelainen S, Lehto S et al. (2004) Gender difference in the impact of type 2 diabetes on coronary heart disease risk. Diabetes Care 7:2898-2904

24. Hu FB, Stampfer MJ, Haffner SM, Solomon CG, Willett WC, Manson JE (2002) Elevated risk of cardiovascular disease prior to clinical diagnosis of type 2 diabetes. Diabetes Care 25:1129 1134

25. Norhammar A, Tenerz A, Nilsson G et al. (2002) Glucose metabolism in patients with acute myocardial infarction and no previous diagnosis of diabetes mellitus: a prospective study. Lancet 359:2140-2144

26. Bartnik M, Ryden L, Ferrari R et al. (2004) The prevalence of abnormal glucose regulation in patients with coronary artery disease across Europe. The Euro Heart Survey on diabetes and the heart. Eur Heart J 25:1880-1890 